\title{
Duckbill-type antireflux self-expandable metal stent placement for post-choledochojejunostomy reflux cholangitis
}

Reflux cholangitis is a common complication of choledochojejunostomy [1,2]. Intestinal content reflux is considered a possible cause for this complication when there is no stenosis at the choledochojejunal anastomosis [3-5], and postcholedochojejunostomy reflex cholangitis (PCRC) is not an indication for endoscopic procedures (e.g., stenting). Here, we discuss using antireflux self-expandable metal stents (ARMS) to treat a patient with PCRC.

A 43-year-old woman who 4.5 years earlier had undergone total pancreatectomy and choledochojejunostomy (Child reconstruction) for multiple pancreatic neuroendocrine tumors presented with fever caused by PCRC. She was treated with antibiotics and ursodeoxycholic acid. However, the fever recurred two or three times a week.

Computed tomography showed pneumobilia, and $99 \mathrm{mTc}-\mathrm{N}$-pyridoxyl-5-methyltryptophan ( $99 \mathrm{mTc}$ TMMT) hepatobiliary scintigraphy showed high tracer uptake in the afferent and blind loops. Endoscopy (CF-H260Al; Olympus Medical Systems, Tokyo, Japan) revealed that the choledochojejunal anastomosis was dilated with cholestasis. No bile duct stones were observed ( $\triangleright$ Fig. 1 ).

We speculated that PCRC was caused by cholestasis in the anastomosis and planned for ARMS placement. Cholangiography showed that the common hepatic duct was $23 \mathrm{~mm}$ in diameter; side-byside placement of two 10-mm ARMS was considered ideal.

A 7-Fr plastic stent (Harmo Ray; Hanaco Medical, Saitama, Japan) was inserted into B6 to prevent the posterior branch from being obstructed by the ARMS. Two duckbill-type ARMS (D-ARMS; $10 \mathrm{~mm} \times$ $6 \mathrm{~cm}$; Kawasumi Laboratories, Tokyo, Japan; Fig. 2) were placed in the anterior branch and the left hepatic duct, respectively, using the side-by-side method. To prevent stent migration, the D-ARMS were fixed to the jejunal mucosa using
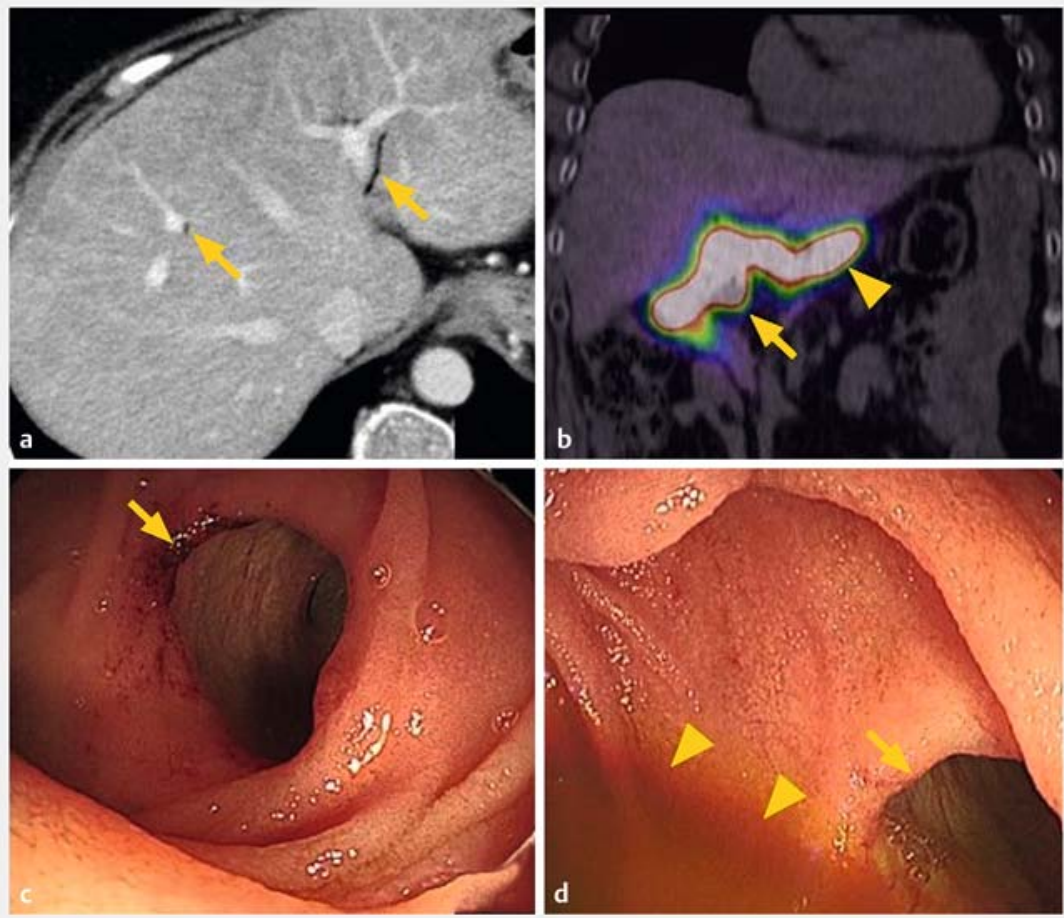

- Fig. 1 Imaging findings before the procedure. a Computed tomography (CT) showing pneumobilia (arrows). b Single-position emission CT (SPECT)/CT 90 min after injection of $99 \mathrm{mT}$-PMT tracer, showing high tracer uptake in the afferent loop (arrow) and blind loop (arrowhead). c, d Endoscopic images showing the choledochojejunal anastomosis markedly dilated (arrows) with cholestasis (arrowheads).
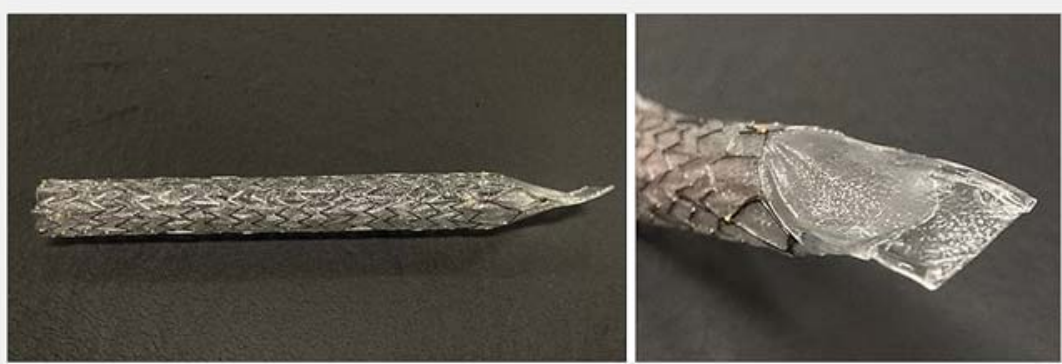

- Fig. 2 Duckbill-type antireflux self-expandable metal stent (D-ARMS).

clips (SureClip; Micro-Tech Endoscopy, Ann Arbor, Michigan, USA) (ฉ Fig. 3, > Fig. 4; V Video 1 ).

Hepatobiliary scintigraphy showed that cholestasis was notably reduced in the afferent and blind loops after the procedure, and the patient's fever had improved ( $\mathbf{F i g} . \mathbf{5}$ )

This is the first report discussing D-ARMS placement in PCRC. Dramatic improve- 

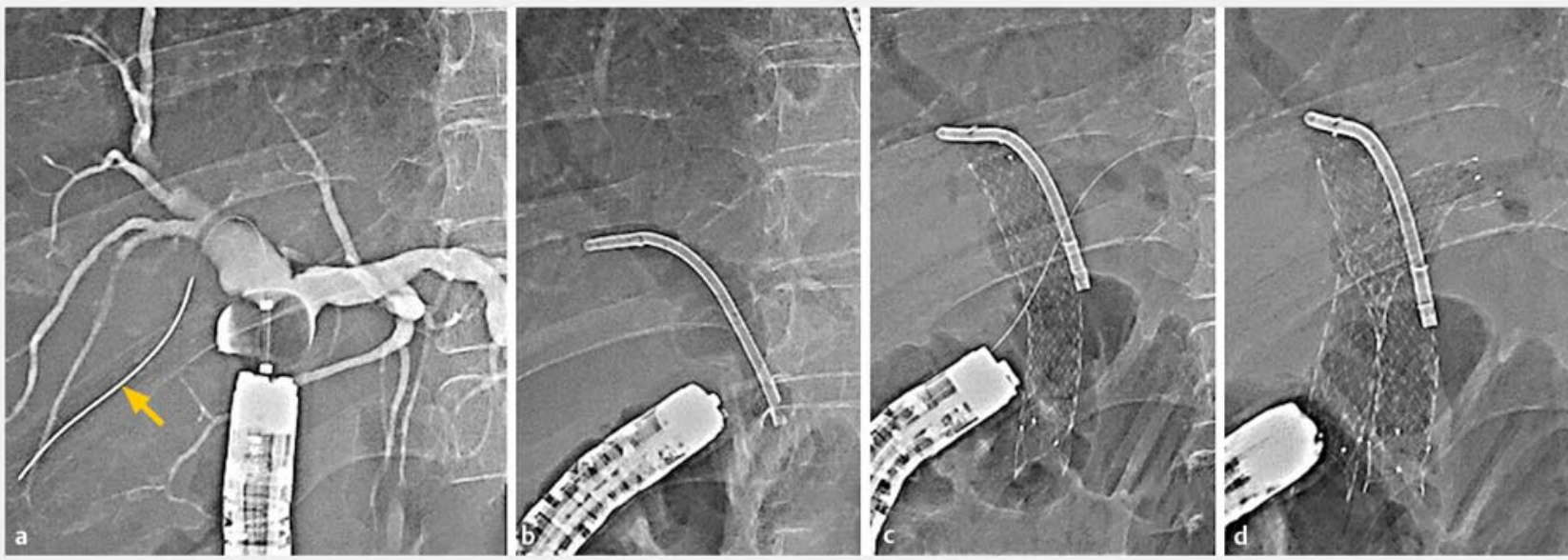

- Fig. 3 Fluoroscopic images of two D-ARMS with plastic stent placement. a Cholangiography with balloon catheter. The guidewire was inserted into B6 (arrow). b A 7-Fr plastic stent was placed in B6. c The first D-ARMS was placed in the anterior branch. $\mathbf{d}$ The second D-ARMS was placed in the left hepatic duct using the side-by-side method.
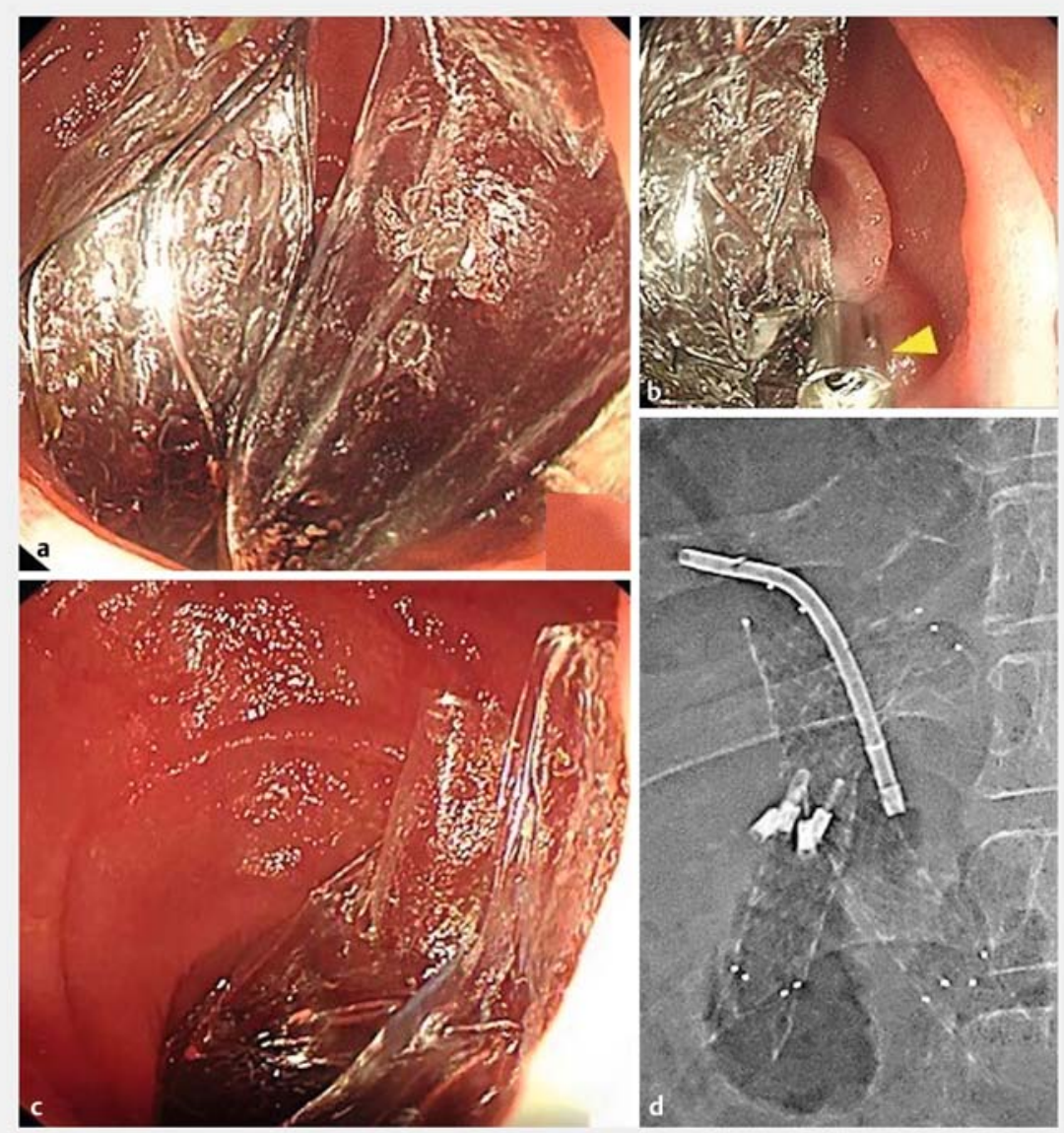

- Fig. 4 Imaging findings after the procedure. a, b Endoscopic images of two D-ARMS on the anastomosis. c The D-ARMS was fixed to the jejunal mucosa using a clip (arrowhead). d Fluoroscopic image after the procedure.

ment of symptoms was observed, deEndoscopy_UCTN_Code_TTT_1AS_2AD
Funding

This work was supported in part by The $\mathrm{Na}$ tional Cancer Center Research and Development Fund (31-A-13).

Competing interests

The authors declare that they have no conflict of interest.

The authors

Takehiko Koga ${ }^{1}$, Susumu Hijioka ${ }^{1}$, Yasutake Ishikawa ${ }^{2}$, Kimiteru Ito ${ }^{3}$, Shota Harai ${ }^{1}$, Takuji Okusaka $^{1}$, Yutaka Saito ${ }^{4}$

1 Department of Hepatobiliary and Pancreatic Oncology, National Cancer Center Hospital, Tokyo, Japan

2 Department of Radiological Technology, National Cancer Center Hospital, Tokyo, Japan

3 Department of Diagnostic Radiology, National Cancer Center Hospital, Tokyo, Japan

4 Department of Endoscopy Division, National Cancer Center Hospital, Tokyo, Japan 

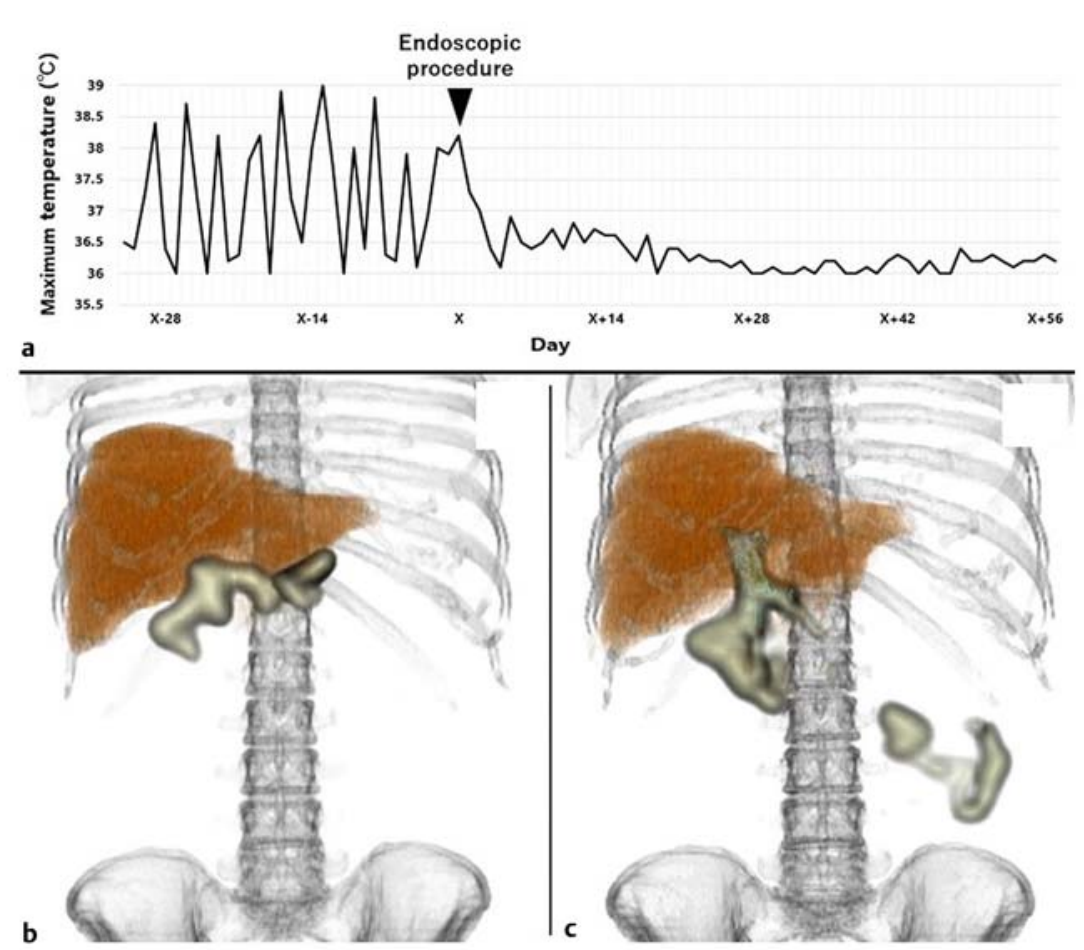

- Fig. 5 Body temperature chart and SPECT/CT images before and after the endoscopic procedure. a Body temperature chart. After the procedure, the fever disappeared completely. b, c Three-dimensional SPECT/CT images ( $90 \mathrm{~min}$ after injection of tracer) before (b) and after the procedure (c). After the procedure, cholestasis was notably reduced in the afferent and blind loops.

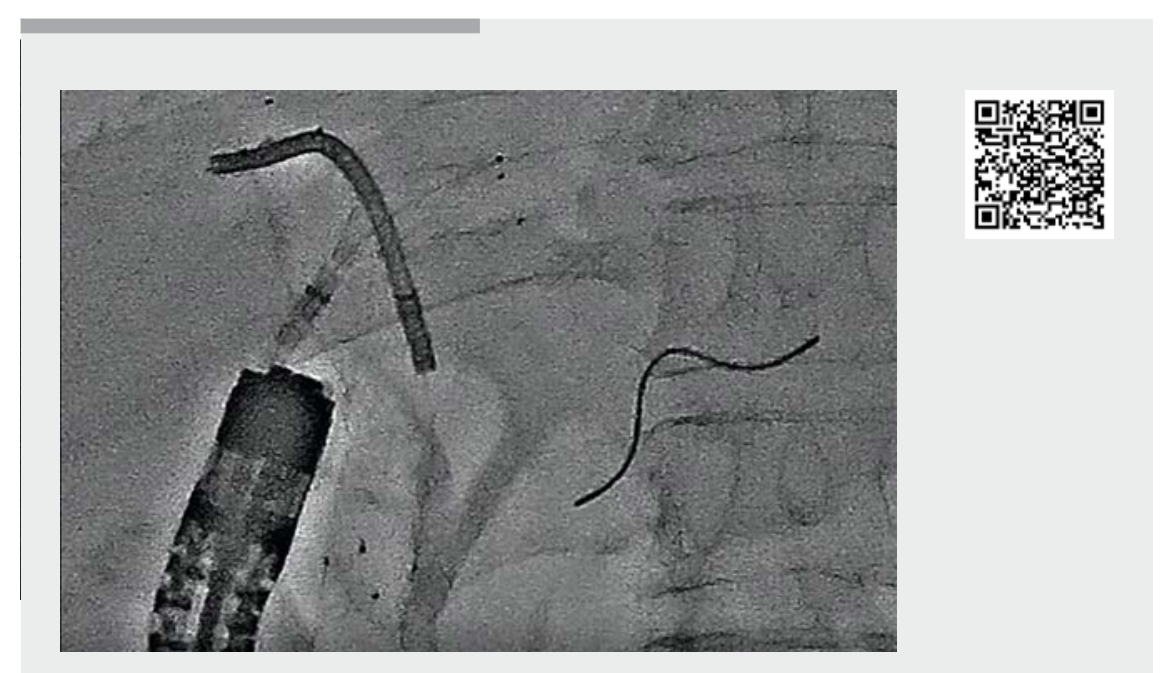

$\checkmark$ Video 1 D-ARMS placement in a patient with refractory post-choledochojejunostomy reflux cholangitis.
Corresponding author

\section{Susumu Hijioka, MD, PhD}

Department of Hepatobiliary and Pancreatic Oncology, National Cancer Center Hospital, 5-1-1 Tsukiji, Chuo-ku, Tokyo 104-0045, Japan

Fax: +81-3-35423815

shijioka@ncc.go.jp

\section{References}

[1] Ueda H, Ban D, Kudo A et al. Refractory longterm cholangitis after pancreaticoduodenectomy: a retrospective study. World J Surg 2017; 41: 1882-1889

[2] Tocchi A, Mazzoni G, Liotta G et al. Late development of bile duct cancer in patients who had biliary-enteric drainage for benign disease: a follow-up study of more than 1,000 patients. Ann Surg 2001; 234: $210-$ 214

[3] Hatanaka H, Yamamoto H, Yano T et al. Double-balloon endoscopy for hepatolithiasis with multiple stones associated with reflux of intestinal contents into the bile duct in a patient with Roux-en-Y hepaticojejunostomy. Dig Endosc 2012; 24: 429

[4] Sanada Y, Yamada N, Taguchi M et al. Recurrent cholangitis by biliary stasis due to non-obstructive afferent loop syndrome after pylorus-preserving pancreatoduodenectomy: report of a case. Int Surg 2014; 99 : 426-431

[5] Yamamoto M, Tahara H, Hamaoka M et al. Utility of hepatobiliary scintigraphy for recurrent reflex cholangitis following choledochojejunostomy: A case report. Int J Surg Case Rep 2018; 42: 104-108

Bibliography

Endoscopy 2021; 53: E174-E176

DOI $10.1055 / \mathrm{a}-1216-1220$

ISSN 0013-726X

published online 20.8.2020

(c) 2020. Thieme. All rights reserved.

Georg Thieme Verlag KG, Rüdigerstraße 14,

70469 Stuttgart, Germany 